\title{
Prevalencia de patologías relacionadas con el asbesto crisotilo en trabajadores de una empresa de productos de crisotilo-cemento
}

\author{
Prevalence of pathological conditions related with \\ chrysotile asbestos in workers of a company that \\ produces chrysotile cement
}

\author{
MAuRIcio MeJía MeJía, MD., ESO. ${ }^{(1) ; ~ I V A ́ N ~ R E N D O ́ N, ~ M D ., ~ M S c . ~}{ }^{(2)}$
}

\section{Resumen}

INTRODUCCIÓN: en Manizales opera una fábrica de productos de fibrocemento que emplea, desde el inicio de sus operaciones, fibras de asbesto crisotilo bajo condiciones de higiene industrial controladas.

OBJETIVO: estudiar la prevalencia de las patologías relacionadas con la exposición a las fibras de crisotilo en el aire en la totalidad de la población activa al 31 de diciembre de 2012, la historia de exposición acumulada a fibras, la antigüedad en la empresa y la historia de tabaquismo.

MATERIALES Y MÉTODOS: estudio de corte transversal en el que se compararon los resultados de las radiografías de tórax acorde con la clasificación Internacional de la Organización Internacional del Trabajo de radiografías de Neumoconiosis con las características etarias, de antigüedad en la empresa, de consumo de tabaco y de exposición acumulada.

RESULTADOS: en todos los trabajadores se determinó ausencia de patología pulmonar o pleural relacionada con la exposición a material particulado.

Se encontró una población con edad promedio de 41 años, antigüedad promedio de 12 años, exposición acumulada a fibras promedio de 1,33 fibras/cc/años, y bajo consumo activo de tabaco.

Al comparar los resultados de este estudio con otros similares disponibles en la literatura, se encontró que lo descrito por otros se replica en esta empresa.

CONCLUSIÓN: no puede descartarse la posibilidad de mantener trabajadores libres de las consecuencias nocivas para su salud por la exposición a las fibras de crisotilo auque se apliquen las medidas de higiene industrial recomendadas.

Palabras clave: asbestos, asbestosis, crisotilo, cáncer pleural, cáncer pulmonar, mesotelioma pleural.

\begin{abstract}
INTRODUCTION: since it began to operate, a factory in the city of Manizales produces fibrocement with chrysotile asbestos bras, under controlled industrial hygiene conditions.

OBJECTIVE: to study the prevalence of pathological conditions related with exposure to chrysotile bras present in the air in the total population active on December 312012 , the history of cumulative exposure to bras, the time of work for the company, and the history of tobacco use.
\end{abstract}

MATERIALS AND METHODS: cross-cut study that compared the results of chest radiographs according to the ILO International Classification of Radiographs of Pneumoconioses, with characteristics of age, time of work for the company, tobacco use, and cumulative exposure.

Esta investigación está adscrita al grupo de Investigaciones en Salud Pública de la Universidad Autónoma de Manizales con el fin de optar al título de Maestría en Salud Pública por el autor principal; el coautor actuó como director de tesis de maestría.

\footnotetext{
${ }^{(1)}$ Medicina Ocupacional, Jefe de Seguridad y Salud en el Trabajo, TopTec SA. Manizales, Colombia.

${ }^{(2)}$ Médico Docente Universidad de Antioquia. Medellín, Colombia.

Correspondencia: Mauricio Mejía Mejía, correo electrónico: mauriciomejiam@ gmail.com

Recibido: 02/07/16. Aceptado: 02/08/16
} 
RESULTS: in all workers, the absence of pulmonary or pleural pathologies associated with exposure to particulate material was determined.

The mean age of the population was 41 years, the mean time of work for the company was 12 years, the mean cumulative exposure to bras was $1,33 \mathrm{bras} / \mathrm{cc} / \mathrm{year}$, and low rates of active tobacco use.

When comparing the results of this study with other studies available in the literature, it was found that has been described in other studies is reproduced in this company.

CONCLUSION: the possibility of keeping workers free from the noxious consequences of exposure to chrysotile bras cannot be ruled out, even when the recommended industrial hygiene measures are applied.

Keywords: asbestos, asbestosis, chrysotile, pleural cancer, lung cancer, pleural mesothelioma.

\section{Introducción}

Desde la antigüedad el hombre ha empleado las fibras de asbestos para su beneficio. Los asbestos son minerales naturales presentes en la corteza terrestre, que tienen propiedades de alta resistencia física, química y térmica, hecho que les confiere relevancia en el uso humano. Recientemente se han empleado agregadas a una matriz aglutinante como elemento que da resistencia a materiales de fibrocemento, fricción y textiles ignífugos (1-4).

Los asbestos son diversos minerales naturales silíceos en forma fibrosa, presentes en la corteza del planeta, que corresponden a silicatos hidratados de algunos metales (magnesio, hierro, calcio, sodio). Existen dos familias de asbestos claramente diferentes por sus características físicas y químicas y por su potencial patogénico $(1-3,5)$. Una de las familias está compuesta por los asbestos anfíboles (fibras rectas, con forma de agujas) de los cuales los más empleados fueron la crocidolita y la tremolita (asbestos azules), y la amosita (asbesto café); éstas son pequeñas fibras rígidas, con forma de agujas, muy resistentes al medio ácido y al calor, que se disgregan en fibras individuales de 100 nanómetros de diámetro. La otra familia corresponde a las serpentinas (fibras curvas, flexibles, encrespadas), de los cuales solo hay un representante, el crisotilo (asbesto blanco), mucho menos resistente al medio ácido y ligeramente menos resistente al calor (5).

El crisotilo está compuesto por dos capas adheridas y enrolladas finamente formando tubos de 25 a 50 nanómetros de diámetro. La capa externa (brucita) está compuesta por dihidróxido de magnesio $\left(\mathrm{Mg}(\mathrm{OH})_{2}\right)$, mientras la interna está compuesta por dióxido de silicio $\left(\mathrm{SiO}_{2}\right)$. El magnesio de la brucita que compone la capa externa, se desintegra en medio ácido (1), lo que permite a los mecanismos de limpieza pulmonar eliminarlo del tejido (disminuyendo significativamente su biopersistencia y, por lo tanto, su potencial patogénico, respecto de los anfíboles) (6).

El potencial patogénico de las fibras (naturales o sintéticas) se ha relacionado con su naturaleza química, dimensiones y biopersistencia. Mientras los asbestos anfíboles presentan en promedio una vida media en el tejido pulmonar de más de 500 días, el crisotilo persiste en promedio 4 días (con un rango de biopersistencia de entre 0,4 y 11 días) (1).

Se considera material en forma de fibras el que presente una relación longitud/diámetro de 3/1 o superior. Es fibra de dimensiones respirables (con potencial patogénico significativo por su capacidad de alcanzar el alvéolo y por la dificultad de salir de él, una vez alcanzado) la que presente diámetro igual o inferior a 3 micras y longitud igual o superior a 5 micras. Se consideran de mayor peligrosidad las fibras con longitud igual o superior a 20 micras (6).

La exposición ocupacional y extraocupacional a altas concentraciones de fibras de asbestos en el aire ha sido relacionada con la aparición de fibrosis pulmonar (asbestosis), cáncer pulmonar, engrosamientos pleurales benignos y mesotelioma pleural maligno (1, 2, 5-10). Las consecuencias de esta 
exposición se reconocieron y documentaron de manera importante a partir de la década de 1930, debido a la exposición intensa derivada, principalmente, del uso altamente difundido de las fibras de asbestos como revestimientos de superficies de estructuras de edificios y buques, con el fin de servir de aislamiento térmico (protección de las condiciones climáticas para las personas que habitan los recintos aislados y protección contra la acción del fuego de las estructuras metálicas de las edificaciones). También se ha observado la aparición de casos de mesotelioma relacionados con exposición a las fibras de asbestos de tipo ambiental (no ocupacional) en Turquía, Chipre y Córcega, y en el área de influencia de depósitos de vermiculita en Montana (EE.UU) que han sido considerados como causados por inhalación de fibras de tremolita (1).

También se han utilizado con otros fines, tales como la confección de textiles y guantes resistentes a altas temperaturas (vestidos para bomberos para combatir el fuego, elementos de seguridad industrial para trabajos con exposición a temperaturas extremadamente altas), aislamientos y empaques de tuberías de conducción de material caliente, fabricación de productos de fibrocemento (tejas onduladas para techos, placas planas para pisos y paredes, donde las fibras de asbesto sirven como apoyo estructural a la matriz y confirieren a las placas mayor resistencia), pastillas y bandas de fricción (frenos y embragues de vehículos), empaques para sistemas de conducción de gases y líquidos calientes, filtros, entre otros $(1,2,4,11)$.

La asbestosis, una forma de neumoconiosis, enfermedad pulmonar no maligna, es una fibrosis parenquimatosa pulmonar que puede ser estática o lentamente progresiva, que puede ir de ligera a grave y llevar hasta la insuficiencia respiratoria y la muerte. Su diagnóstico se fundamenta frecuentemente en los hallazgos clínicos y la historia de exposición ocupacional significativa al material, con un prolongado período de latencia entre la exposición y la enfermedad (usualmente igual o superior a 10 o 15 años), en la observación de opacidades irregulares en la radiografía del tórax y en la capacidad de difusión pulmonar inferior a lo normal.
La confirmación diagnóstica se hace por anatomía patológica, en la que se observan los cuerpos de asbestos y la fibrosis pulmonar $(1,5,6)$.

Las alteraciones pleurales benignas relacionadas con los asbestos son los engrosamientos y placas pleurales, la fibrosis pleural difusa y los derrames pleurales benignos. En un paciente en particular pueden encontrarse una o cualquier combinación de estas alteraciones. No está claro el mecanismo que genera las alteraciones pleurales benignas, pero se ha considerado que puede tener relación con la traslación de fibras de asbestos desde el parénquima pulmonar al espacio pleural donde causan una reacción inflamatoria en la superficie de la pleura parietal $(1,5,6)$.

El mesotelioma maligno es una enfermedad progresiva que puede afectar la pleura, el peritoneo, el pericardio o la túnica vaginalis. Es una entidad de muy mal pronóstico; el tumor pleural crece sin parar hasta comprimir el pulmón y las estructuras mediastinales. Las metástasis distales son raras $(1,5,6)$.

El cáncer pulmonar en los expuestos a los asbestos es similar al cáncer pulmonar de los no expuestos. Se considera que el cáncer obedece a un daño en el ADN celular, y es dependiente de la extensión y de la naturaleza del daño celular, pueden ser de cualquier tipo de células y, desde el punto de vista patológico es indiferenciable del cáncer atribuible al humo de tabaco en fumadores $(1,5,6)$.

Debido a la aparición de casos de patologías respiratorias en trabajadores que, por motivo de su trabajo, tuvieron exposición a fibras de asbestos, surge una tendencia mundial hacia la búsqueda de nuevos casos y hacia el ataque contra toda forma de uso de los minerales englobados dentro de la clasificación de "asbestos". A medida que avanzaron las investigaciones y aumentaron dramáticamente los reportes de enfermos graves y fatales considerados como consecuencia de la exposición laboral a fibras de asbestos en el aire, nació una amplia, intensa y apasionada controversia respecto del nivel de riesgo de enfermar para los diferentes trabajadores expuestos bajo distintos grados de exposición y a los diversos materiales que conforman la familia de los asbestos $(8,12,13)$. 
La atención sobre el riesgo para la salud derivado de los asbestos se inició en 1964 cuando Selikoff (14), del hospital Monte Sinaí, Nueva York, publicó un artículo acerca de la elevada frecuencia de mesotelioma observada en trabajadores de aislamiento de Nueva York expuestos a asbestos (15). En dicho país, en 1976, en respuesta a la propuesta de la EPA (Environmental Protection Agency, agencia estatal de protección ambiental estadounidense) de prohibir el uso de los asbestos, el congreso emitió el acta de control de sustancias tóxicas ("Toxic Substances Control Act”, TSCA) con la intención de regular las sustancias químicas tóxicas diferentes de los pesticidas, en la que se incluyeron los asbestos. A partir de entonces, se generó una polémica controversia que llevó a la prohibición del uso de los asbestos en 1989, (manufactura, importación, procesamiento y distribución de todos los productos con asbestos), efectiva a partir de 1997. Inicialmente, la decisión de prohibir todos los usos de todos los asbestos fue denegada en las altas cortes norteamericanas, por falta de análisis de los riesgos de los sustitutos y de las implicaciones económicas de la prohibición de todos los usos de las sustancias ampliamente empleadas. Esta decisión llevó a la EPA a prohibir los nuevos usos de los asbestos a partir de 1994. La controversia se desvió hacia el interés público de retirar los asbestos instalados como aislante térmico en los techos de las escuelas norteamericanas (controversia que incluyó el análisis del costo y riesgo de las tareas requeridas para el desmonte del asbesto como aislante de todos los edificios públi$\cos$ ), en respuesta a la inmensa publicidad dada al debate del riesgo del uso de las fibras (15). Finalmente, se consideró más riesgoso retirar los asbestos de su sitio instalado que dejarlos en el mismo, al no encontrar contaminación significativa en el aire de las habitaciones aisladas, donde la concentración observada no fue significativamente diferente en el interior de los edificios aislados con asbestos, que en el interior de los edificios que no los contenían y que en el aire ambiente externo (16).

Un factor de confusión muy importante es la relación entre fumar y el nivel de riesgo del cáncer relacionado con los asbestos, debido a la sinergia entre ambos (15). Por otro lado, Powell (15) menciona que la falta de consistencia en los análisis y toma de decisiones en Estados Unidos tuvo como fundamento la ignorancia de los tomadores de decisiones y la carencia de científicos expertos en el tema al interior de las agencias gubernamentales, de manera que las mismas debieron acudir a contratistas externos, presentándose un importante sesgo al momento de decidir el paso a seguir $(9,10)$.

A partir de la década de 1950 la ACGIH (American Conference of Governmental Industrial Hygienists, asociación independiente de higienistas industriales gubernamentales, de norteamérica) recomendó un límite máximo permisible para la concentración ambiental de las fibras de asbesto en el aire respirado por los trabajadores, iniciando en 5 millones de partículas por pie cúbico (aproximadamente 177 fibras por centímetro cúbico). En 1972 la OSHA (Occupational Safety and Health Agency, agencia norteamericana de Salud y Seguridad Ocupacional) estableció el límite legal de concentración ambiental en 5 fibras por centímetro cúbico. Finalmente, en 1994 estableció el límite de 0,1 fibras por centímetro cúbico (15), valor vigente en la actualidad.

En Italia se prohibieron todos los procesos productivos relacionados con todos los asbestos a partir de 1992, como resultado de los hallazgos en la salud de los trabajadores expuestos durante todo el siglo (18), los cuales no se diferencian de lo observado en otros países subtropicales. En 1999 una directiva de la Unión Europea prohibió la comercialización y uso de cualquier tipo de asbesto a partir de diciembre de 2002 (6).

En Latinoamérica se prohibió el uso de los asbestos en todas sus formas en Argentina, Chile y Uruguay en 2001 y 2002 en un escenario de presiones políticas internacionales hacia la prohibición de la explotación y uso de todos los asbestos (7), mientras que otros países han tomado decisiones contra la prohibición total y en favor del uso controlado $(3,4,11,17,19)$.

Se encuentran abundantes escritos en la literatura que mencionan los peligros de la exposición humana a los asbestos de manera general, siempre apoyados 
en las consecuencias en la salud de los trabajadores expuestos masivamente, ante todo en las regiones subtropicales del hemisferio norte $(18,20,21)$. En todos ellos se encuentra como característica común la ausencia de diferenciación entre los diferentes tipos de asbestos, la generalización del uso y de las circunstancias de exposición a las condiciones vividas en la primera mitad del siglo XX en Europa y en los Estados Unidos de Norteamérica. Sin embargo, se encuentran también estudios publicados en los que se menciona la ausencia de enfermedad entre trabajadores que emplean el crisotilo como materia prima en procesos productivos en los que se cuenta con controles de higiene ocupacional, en trabajadores sometidos a programas de vigilancia epidemiológica estrictos que permiten confiar en la valoración de la efectividad de los controles concluida a partir de ellos $(4,11,18,21,22)$.

La controversia descrita se fundamenta en dos puntos básicos; el primero se trata de la severidad del daño en la salud por la exposición laboral a fibras respirables de asbestos antes de conocerse el peligro de estos materiales, en trabajadores que laboraban bajo condiciones de exposición a concentraciones masivas, sin ningún tipo de medida de control ambiental ni personal, con graves consecuencias en su salud documentadas posteriormente. Esta condición contrasta con la manipulación de los mismos materiales en momentos posteriores a la identificación del riesgo, llevada a cabo mediante la aplicación de medidas de higiene industrial que permitieron reducir significativamente las concentraciones de material en el aire del ambiente de trabajo, y mediante el uso de elementos de protección personal $(22,23)$. El segundo punto que fundamenta la controversia es el hecho de que los dos tipos de fibras de asbestos, los anfíboles y las serpentinas, tienen diferentes niveles de peligro, así como la existencia de productos empleados como sustitutos para los asbestos, que mientras unos los consideran seguros, otros no $(1-3,5,12)$.

En el contexto de esta discusión surgen algunos estudios epidemiológicos que buscan evaluar el comportamiento en campo de las patologías consideradas como producidas por la exposición a los asbestos en trabajadores expuestos exclusivamente a crisotilo, tales como la fibrosis pulmonar, el cáncer pulmonar y el mesotelioma pleural $(22,23)$. Este estudio de investigación busca observar el comportamiento epidemiológico de las patologías consideradas como relacionadas con la exposición a asbestos, en la población trabajadora de TopTec SA., fábrica de productos de fibrocemento, que ha empleado fibras de asbesto crisotilo (sin anfíboles) bajo condiciones de higiene industrial controladas desde su creación en 1981 hasta el momento del corte del estudio, en la totalidad de sus trabajadores activos al momento del corte del estudio, con la intención de aportar información actual del país a la discusión técnica y política respecto de la pertinencia de optar por la prohibición de cualquier uso del asbesto crisotilo y emplear otro tipo de fibras, o la posibilidad de preferir un enfoque preventivo para el uso, tanto del asbesto crisotilo como de otras fibras usadas como sustituto, de forma controlada.

La empresa Tecnología en Cubrimiento, TopTec SA., donde se desarrolló el estudio, contaba con 183 trabajadores a diciembre de 2012, con más de un año de antigüedad, al momento del corte del estudio. Las materias primas utilizadas fueron asbesto crisotilo, cemento, carbonato de calcio, bentonita, sílice, celulosa y agua.

\section{Materiales y métodos}

Estudio de corte transversal desarrollado con la totalidad de los trabajadores activos al 31 de diciembre de 2012 con más de un año de antigüedad de la empresa de materiales de fibrocemento TopTec SA., dirigido a establecer la prevalencia de las enfermedades que se han considerado relacionadas con la exposición a asbestos y a observar si se encuentra alguna diferencia en la prevalencia de la enfermedad entre las diferentes cohortes seleccionadas según niveles de exposición acumulada, en relación con el tiempo de exposición y con la historia de consumo de tabaco.

La investigación se consideró sin riesgo ético y fue aprobada por el Comité de Ética de la Universidad Autónoma de Manizales. 
La población objeto del estudio fueron todos los trabajadores activos al 31 de diciembre de 2012 en la empresa y que hubieran laborado más de un año.

Se analizó el número de años de exposición a crisotilo, la exposición acumulada a crisotilo y el número de años de trabajo, todos durante la permanencia en la empresa. Se indagó y tabuló lo reportado por el trabajador en cuanto a los antecedentes de exposición a asbestos en otras empresas durante toda la vida laboral.

Se consideraron como variables sociodemográficas la edad al momento del corte del estudio y la edad al momento del diagnóstico de las patologías en estudio.

Se consideró el hábito de fumar, respecto del cual se registró el número de años de consumo de tabaco, número de paquetes/año consumidos, tiempo de abandono del hábito al momento del corte del estudio (31 de diciembre de 2012) y tiempo de abandono del hábito al momento del diagnóstico de las patologías en estudio.

Como variable dependiente se consideró el reporte de lectura de la radiografía de tórax con metodología de la Organización Internacional del Trabajo (OIT) para neumoconiosis. Los casos de lecturas 1/0 o menores, se consideraron negativos. Los casos de lectura 1/1 o mayor y/o presencia de placas pleurales, se consideraron sospechosos. Para estos últimos se planteó como variable dependiente el resultado de las pruebas confirmatorias para las patologías relacionadas con el asbesto, tales como resultado de tomografía computarizada de alta resolución (TACAR), toracoscopia, broncoscopia, biopsia pleural o pulmonar, o anatomía patológica, de acuerdo con lo realizado.

La información se recolectó a partir de los registros de historias clínicas ocupacionales, de los registros de talento humano y de los registros de mediciones ambientales de fibras, y se registraron en una base de datos de Excel que consolidó los resultados de los paraclínicos practicados (radiografías de tórax con lectura acorde con la metodología para lectura de placas para neumoconiosis de la OIT) y los resultados de la exposición acumulada a fibras.

\section{Resultados}

No se encontró ningún caso de trabajadores que cursara con las patologías en estudio (no se realizó análisis bivariado entre las variables de interés al encontrar valor de cero para la variable dependiente en todas las series de trabajadores).

Se encontraron 183 trabajadores que cumplieron con los criterios de inclusión (haber trabajado durante un año o más, y estar activos al 31 de diciembre de 2012).

A los 183 trabajadores de la empresa evaluados se les realizaron radiografías de tórax periódicas acordes con las técnicas establecidas en la clasificación de la OIT para radiografías de tórax para neumoconiosis, y todos tuvieron control radiográfico en 2012. Todas las radiografías fueron leídas por dos médicos: un radiólogo y médico ocupacional. Todas las lecturas de las radiografías se reportaron sin evidencias de cambios de neumoconiosis. No hubo ningún caso con hallazgos radiográficos sospechosos de las enfermedades objeto del estudio.

Los 183 trabajadores tenían edades entre los 20 y los 60 años, con un promedio de 41,14 años y desviación estándar de 9,93 años. La mayoría se hallaba entre los 25 y los 54 años (en este rango de edad se encontraron 157 individuos, lo que corresponde al $86 \%$ de la población) (figura 1).

La antigüedad de los 183 trabajadores estuvo comprendida entre los 1 y los 30 años, con un

\section{Distribución por edad}

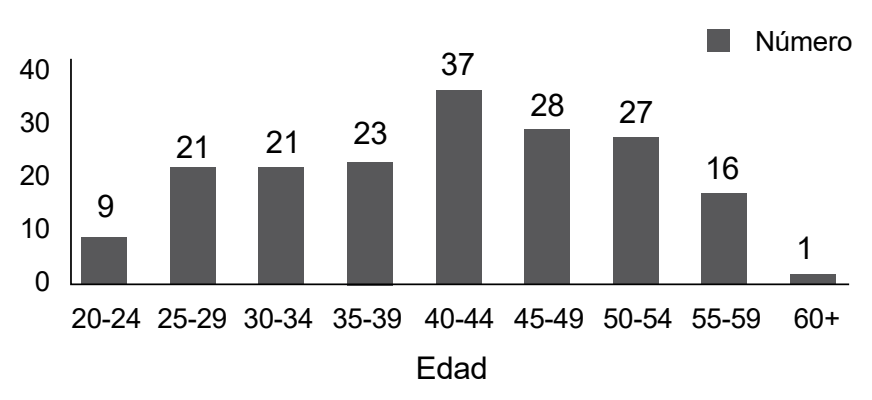

Figura 1. Distribución de trabajadores dentro de los rangos de edad por quinquenios.

Revista Colombiana de Neumología Vol 28 Nº 2 | 2016 
promedio de 12,47 años y una desviación estándar de 8,34 años. Con antigüedad superior a 10 años se encontraron 93 individuos (50,8\% de la población), en tanto que con antigüedad superior a 20 años se hallaron 44 (24\% de la población) (figura 2).

Los 183 trabajadores se encontraron con sumatoria de exposición acumulada de entre un valor mínimo de 0,0 y un valor máximo de 24,96 fibras/ml/años, con un promedio de 1,33 fibras/ml/años y una desviación estándar de 3,1 fibras/ml/años. Se dividieron todos los trabajadores en tres cohortes por grupos de exposición acumulada. En la primera se agruparon todos los trabajadores con niveles de exposición acumulada a fibras inferior a $1 \mathrm{fibra} / \mathrm{ml} / \mathrm{años}$, en la segunda se agruparon todos los trabajadores con niveles de exposición acumulada a fibras igual 1 y menor a 10 fibras/ $\mathrm{ml} / \mathrm{años}$ y en la tercera se agruparon todos aquellos con niveles de exposición acumulada a fibras igual o superior a 10 fibras/ml/años. La mayor frecuencia se encontró por debajo de $1 \mathrm{fibra} / \mathrm{ml} / \mathrm{años}$, grupo que comprende a 116 trabajadores $(63,4 \%)$. Entre 1 y 9,99 fibras/ml/años se encontraron 63 trabajadores $(34,4 \%)$ y con exposición acumulada de 10 fibras $/ \mathrm{ml} /$ años o más se encontraron 4 trabajadores $(2,2 \%)$.

Entre los trabajadores con antigüedad entre 10 y 19 años se encontró un promedio de exposición acumulada a 1,11 fibras/cc/años, con un mínimo de $0,0 \mathrm{fibras} / \mathrm{ml} / \mathrm{años} \mathrm{y} \mathrm{un} \mathrm{máximo} \mathrm{de} 7,657 \mathrm{fibras} / \mathrm{ml} /$ años. Entre los trabajadores con antigüedad de 20 años en adelante se encontró un promedio de exposición acumulada a 3,781 fibras/ml/años, con un mínimo de 1,362 fibras/ml/años y un máximo de 24,963 fibras/ml/años (figura 3).

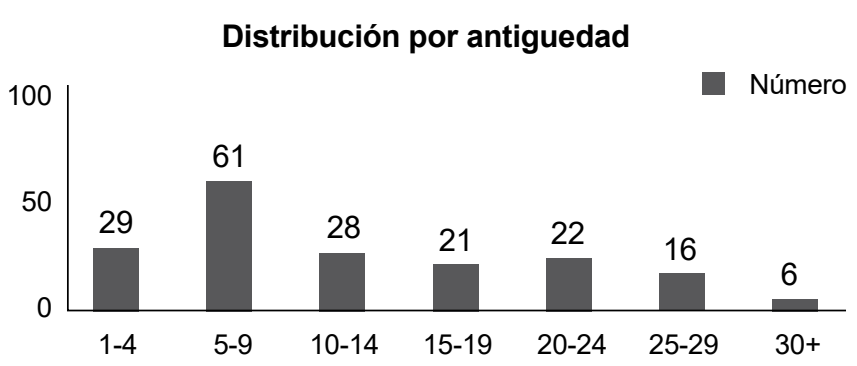

Figura 2. Distribución de trabajadores dentro de los rangos de antigüedad por quinquenios.
No hubo ningún caso en el que el trabajador informara antecedentes ocupacionales en los que se pudiera sospechar la exposición a otros agentes neumoconióticos.

De los 183 trabajadores se encontraron $119(65 \%)$ sin historia de consumo de tabaco.

Se encontraron 64 trabajadores (35\%) con historia de tabaquismo, los cuales han fumado entre $1 \mathrm{y}$ 45 años, con una media de 4 años y una desviación estándar de 7,88 años. 37 trabajadores $(20,2 \%)$ han fumado entre 1 y 4 años, 21 trabajadores $(11,5 \%)$ han fumado entre 5 y 9 años, 4 trabajadores $(2,2 \%)$ han fumado entre 10 y 14 años, un trabajador $(0,55 \%)$ ha fumado entre 15 y 19 años, y un trabajador $(0,55 \%)$ ha fumado más de 20 años (figura 4).

Entre los fumadores se encuentran 10 trabajadores $(5,5 \%)$ fumadores activos. Hay 54 exfumadores $(29,5 \%)$, con un promedio de 3 años de tiempo desde la suspensión del hábito, con desviación estándar de 6,91 años desde la suspensión del consumo.

Los fumadores cuentan con un consumo acumulado promedio de 1,73 paquetes/año y una desviación estándar de 5,42 paquetes/año, con un mínimo de 0,05 y un máximo de 40 paquetes/año. Se encontraron 23 trabajadores con consumo acumulado inferior a 1 paquete/año, 33 trabajadores con consumo acumulado entre 1 y 10 paquetes/año, 5 trabajadores con consumo acumulado entre 11 y 20 paquetes/año, un trabajador con consumo acumulado entre 21 y 30 paquetes/año, y dos trabajadores con consumo acumulado entre 31 y 40 paquetes/año (figura 5).

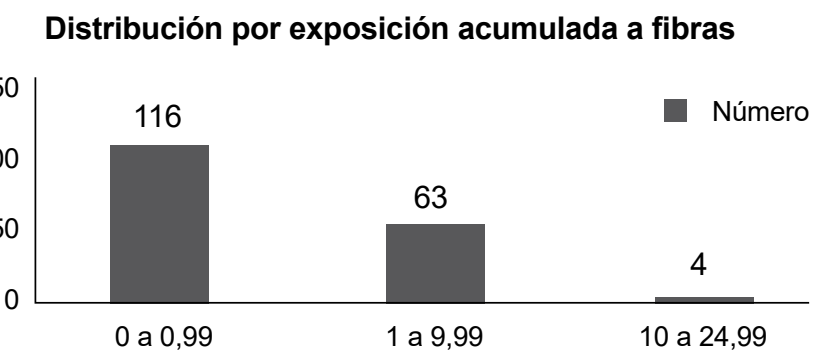

Figura 3. Distribución de trabajadores por exposición acumulada a fibras (fibras/ml/años). 


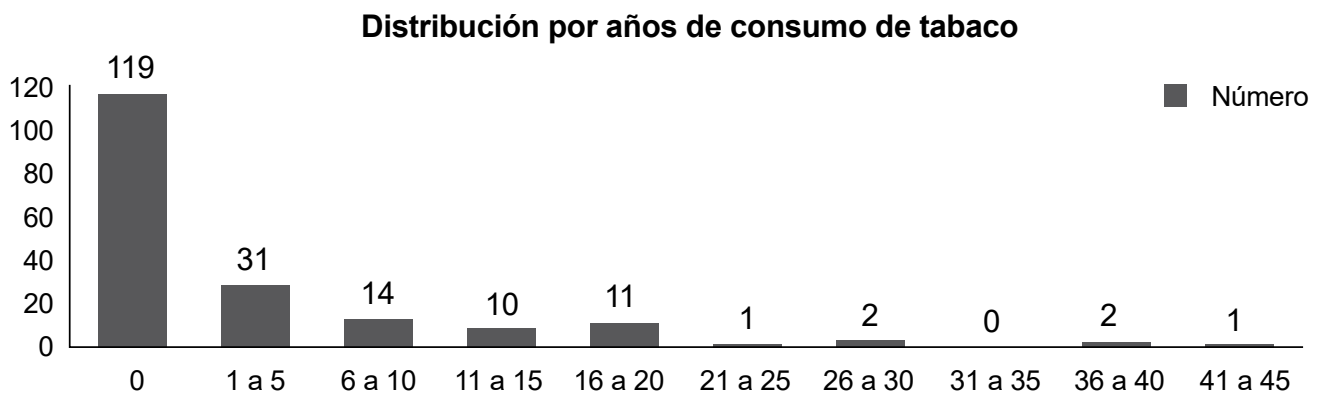

Figura 4. Distribución de trabajadores por número de años de consumo de tabaco, en grupos quinquenales.

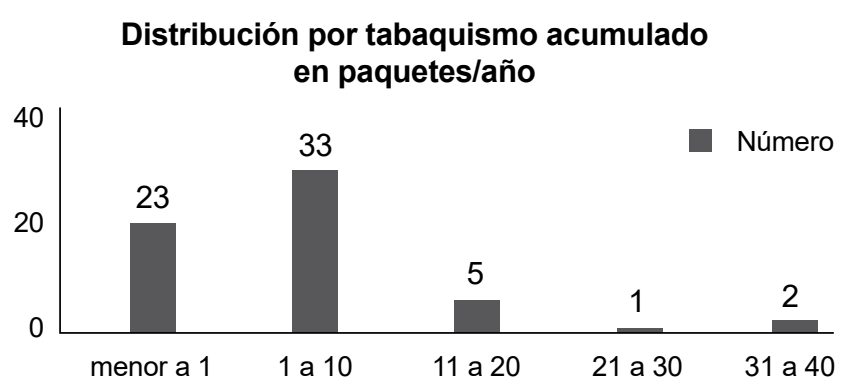

Figura 5. Distribución de trabajadores por consumo acumulado de tabaco, en paquetes/año.

\section{Discusión}

Se trató de una población trabajadora estable, con baja rotación y alta antigüedad. La exposición acumulada a fibras de crisotilo mostró unos ambientes de trabajo con exposiciones controladas en la mayoría de los puestos de trabajo, con algunas excepciones en las que se reportaron concentraciones de fibras por encima de los límites permisibles, mediciones elevadas que no persistieron, y a partir de las cuales algunos trabajadores acumularon una exposición cercana a $25 \mathrm{fibras} / \mathrm{ml} / \mathrm{años}$. Sin embargo, a pesar de estos casos aislados de alta exposición acumulada (los trabajadores que cuentan con exposición acumulada superior a 10 fibras/ $\mathrm{ml} /$ año tienen una antigüedad superior a 20 años) y considerando que el promedio de antigüedad supera el umbral del período de latencia de 10 años descrito en la literatura (1) para observar los cambios radiográficos en las patologías derivadas de la exposición a fibras en el aire, no se identificaron cambios sugestivos de daño pulmonar relacionado con la exposición en ninguno de ellos. Algunos autores consideran un período de latencia de hasta 20 años (5) para exposiciones en ambientes controlados; el estudio contó con un importante número de trabajadores en la serie analizada con este nivel de antigüedad (44 trabajadores, 24\% de la población), en los que no se hicieron hallazgos sugestivos de las patologías objeto del estudio. Estos trabajadores tuvieron un promedio de exposición acumulada a fibras de 3,781 fibras/ml/años, con un valor mínimo de $1,362 \mathrm{fibras} / \mathrm{ml} / \mathrm{años}$ y un valor máximo de 24,963 fibras/ml/años.

Respecto del tabaquismo se reportaron 119 trabajadores (65\%) sin historia de consumo de tabaco, 54 exfumadores $(29,5 \%)$ y 10 fumadores activos $(5,5 \%)$. Los fumadores tuvieron un consumo acumulado de tabaco promedio de 1,73 paquetes/año. Al comparar la prevalencia actual del consumo de tabaco en la empresa $(5,5 \%)$ con la población general, se encontró un valor muy por debajo de lo reportado para la población colombiana del $23,5 \%$ para hombres y $21 \%$ para mujeres (24) (el 93,6\% de los trabajadores de la empresa son hombres y el $6,4 \%$ mujeres). En TopTec SA. se llevan a cabo actividades periódicas de capacitación y fomento contra el consumo de tabaco y se prohíbe su consumo al interior de la institución. La diferencia en el consumo activo actual en la empresa y la tasa de exfumadores $(29,5 \%)$, podría suponerse relacionada con las actividades que se han desarrollado con el fin de desmotivar su consumo. 
Los procesos productivos de TopTec SA. se han desarrollado desde el inicio de las actividades productivas, en 1981, y hasta la fecha del corte del estudio, empleando fibras de asbesto, exclusivamente crisotilo, manteniendo los puestos de trabajo bajo condiciones controladas a través de medidas de higiene industrial tales como sistemas de control local por aspiración (en los lugares donde se ingresan las fibras al proceso productivo y en la transformación de placas) y mediante la operación en húmedo en el resto de procesos. Se ha comprobado la efectividad del control mediante mediciones periódicas (anuales y semestrales) de las concentraciones de fibras en el aire del ambiente de trabajo, con lo que se ha evidenciado que el nivel de concentración de las fibras se ha mantenido, con dos excepciones, en la totalidad de los puestos y en la totalidad de las mediciones, por debajo del límite permisible. Se han empleado elementos de protección certificados para uso en ambientes con fibras de asbestos para controlar los riesgos derivados de posibles fallos en las máquinas, en los procesos o por errores de los trabajadores.

Es posible concluir que, en el caso de los trabajadores activos de TopTec SA., a la fecha del corte del estudio, las medidas de higiene ocupacional implementadas desde el inicio del proceso productivo de la empresa, tales como uso de un mineral considerado por algunos como menos riesgoso, así como su uso en condiciones de ambientes controlados a través de las medidas recomendadas por los expertos (métodos de ventilación local por aspiración, métodos de trabajo húmedos) han permitido contar con una población trabajadora activa libre de patologías relacionadas con la exposición a las fibras de la materia prima. Por otro lado, las medidas adicionales implementadas, tales como la educación para la prevención respiratoria y el control del hábito del tabaquismo, podrían estar complementando las actividades de prevención $y$, por lo tanto, contribuyendo a los resultados positivos encontrados.

Al comparar los resultados de este estudio con otros similares disponibles en la literatura, se encuentra que lo descrito por autores como Sichletidis et al. (22), y Newhouse y Sullivan (23) se repite en TopTec. Estos resultados permiten concluir que no puede descartarse la posibilidad de mantener trabajadores libres de las consecuencias nocivas para su salud derivados de la exposición a las fibras de asbesto crisotilo cuando se desarrollan las actividades laborales bajo la aplicación de las medidas de higiene industrial recomendadas.

Como corolario puede mencionarse que el estudio aporta a los conceptos de trabajo seguro, apoyando al criterio de que los peligros son generadores de riesgos en la medida en que no se controlen y que los riesgos pueden prevenirse mediante la aplicación de los métodos de trabajo seguro.

Se recomienda continuar las labores en la empresa donde se desarrolló el estudio bajo las medidas de higiene industrial consideradas efectivas para prevenir la aparición de enfermedades respiratorias relacionadas con la exposición a material particulado en el aire. Así mismo, se recomienda mantener en aplicación las medidas actuales de vigilancia epidemiológica ocupacional respiratoria con el fin de garantizar el diagnóstico precoz de cualquier patología posiblemente relacionada con la exposición a material particulado en el aire, en cuyo caso se propone intervenir la exposición de manera inmediata para evitar la aparición de nuevos casos.

Se sugiere, adicionalmente, ampliar la presente investigación mediante la evaluación del estado de salud de los trabajadores retirados y que hayan trabajado un período de tiempo significativo en la empresa, con el fin de confirmar o descartar la presencia de algún caso de patología no reportado que pudiera tener relación con la exposición a las fibras de crisotilo durante su actividad laboral en la empresa.

\section{Financiación y conflictos de interés}

Para el desarrollo de la investigación no se contó con ningún tipo de auspicio económico; todos los costos de la investigación fueron asumido por el autor principal.

El programa de vigilancia epidemiológica respiratoria de la empresa donde se desarrolló el estudio se lleva a cabo con apoyo de la ARL, aseguradora del Sistema General de Riesgos Laborales, con autonomía de la empresa. 
El autor principal ejerce la Medicina Ocupacional en la empresa objeto del estudio, por lo que sus observaciones podrían estar sesgadas por su visión interna de la empresa.

Las lecturas radiográficas son elaboradas en primera instancia por un Médico Radiólogo vinculado a una IPS de radiología externa, no vinculada de ninguna manera a la empresa donde se desarrolla el estudio. Los costos de las radiografías hechas dentro de los exámenes periódicos y la gestión administrativa de su realización fueron asistidos por la entidad aseguradora del Sistema General de Riesgos Laborales a la que están afiliados los trabajadores.

El coautor es un Médico del ámbito académico que no presenta vínculo de ningún tipo con la empresa en la que se desarrolla el estudio, por lo que no declara ningún conflicto de interés.

\section{Bibliografía}

1. Baxter PJ, Aw TCh, Cockcroft A, Durrington P, Harrington JM. Hunter's diseases of occupations. 9th. ed. Londres: Ed. Arnold; 2000. p. 686-96.

2. Tossavainen A. El asbesto en el mundo: producción, uso e incidencia de las enfermedades relacionadas con el asbesto. Ciencia y Trabajo. 2008(27):7-13.

3. Albuquerque H.. Aspectos sobre la producción del amianto, exposición y vigilancia de los trabajadores expuestos al amianto en Brasil. Ciencia y Trabajo 2008(27):11-7.

4. Mujica N, Arteta JM. Asbesto en Venezuela. Ciencia y Trabajo 2008(27):21-4.

5. Martínez C, Quero A, Isidro I, Rego G. Enfermedades pulmonares producidas por inhalación de polvos inorgánicos. La Medicina Hoy. s. f. 2001. [Acceso 31 Jul 2014] Disponible en http://test.elsevier.es/ficheros/sumarios/1/61/1397/34/1v61n1 397a13016709pdf001.pdf)

6. G Luis, C Hernández, C Rubio, I Frias, A Gutiérrez, A Hardisson. Toxicología del asbesto. Cuad Med Forense. 2009; 15(57):207-13.

7. Contreras G. Enfermedades pulmonares relacionadas con la exposición a asbesto. Cienc Trab. 2006;8(21):117-21.

8. Algranti E. Editorial. El Simposio Latinoamericano del Asbesto (ASLAC). Ciencia y Trabajo. 2008(27):A1.

9. Yarborough C. The risk of mesothelioma from exposure to chrysotile asbestos. Curr OP Pulmonary Med. 2007;13(4):334-38.
10. Yarborough C. Chrisotile as a cause of mesothelioma: an assessment based on Epidemiology. Crit Rev Toxicol. 2006;36(2):165-87.

11. Prieto S. Ciencia y Trabajo 2008(27):18-20. Algunas consideraciones sobre el uso del asbesto en la república de Cuba. Ciencia y Trabajo 2008(27):18-20.

12. Attaran A, Boyd DR, Stanbrook MB. Asbestos mortality: a Canadian export. CMAJ. 2008;179(9):871-2.

13. Banks DE, Shi R, McLarty J, Cowl CT, Smith D, Tarlo SM, et al. American College of Chest Physicians Consensus Statement on the Respiratory Health Effects of Asbestos. Results of a Delphi Study. FCCP. CHEST 2009;135(6):1619-27.

14. Selikoff IJ, Churg J, Hammond C. Asbestos Exposure and Neoplasia. JAMA. 1964;188(1):22-6.

15. Powell MR. The 1983-84 Suspensions of EDB under FIFRA and the 1989 Asbestos Ban and Phase out Rule under TSCA: Two Case Studies in EPA's Use of Science. Discussion Paper. Resources for the Future March 1997.

16. Crump KS, Farrar DB. Statistical analysis of data on airborne asbestos levels collected in an EPA survey of public buildings. Regulatory Toxicology and Pharmacology 1989;10(1):51-62.

17. Harari R. El asbesto en Ecuador: el día después. Ciencia y Trabajo 2008(27):25-30.

18. Marsili D, Comba P, Bruno C, Calisti R, Marinaccio A, Mirabelli $\mathrm{D}$, et al. La prevención de las patologías del asbesto: perspectivas operativas de la cooperación italiana con los países de América latina. Rev Salud Pública. 2010;12(4): 682-92.

19. Reglamento de higiene y seguridad del crisotilo y otras fibras de uso similar. Resolución 007 del 2 de noviembre de 2011. Ministerio de Salud y Protección Social de Colombia.

20. Marsili D. La globalización del riesgoso asbesto. Cooperación Científica Italia Ecuador. La Prevención de la Patología del Amianto: un Problema de Salud Pública. Quito, Roma, 2009;43:31-55.

21. Berry G. Mortality and cancer incidence of workers exposed to chrysotile asbestos in the friction-products industry. Ann Occup Hyg. 1994;38(4):539-46.

22. Sichletidis L, Chloros D, Spyratos D, Haidich AB, Fourkiotou I, Kakoura M, et al. Mortality from occupational exposure to relatively pure chrysotile: a 39-year study. Respiration. 2009;78:63-8.

23. Newhouse ML, Sullivan KR. A Mortality study of workers manufacturing friction materials. $\mathrm{Br} \mathrm{J}$ Ind Med. 1989;46:176-9.

24. Valdes-Salgado R, Hernández-Ávila M, Sepúlveda-Amor J. El consumo de tabaco en la región americana: elementos para un programa de acción. Salud Pública Mex. 2002;44(1):125-35. 